\title{
Low carbon energy behaviors in the workplace: A qualitative study in Italy and Spain
}

\author{
Adina Dumitru $^{a}$, Eugenio De Gregorio ${ }^{d}$, Mirilia Bonnes ${ }^{c}$, Marino Bonaiuto ${ }^{c}$, \\ Giuseppe Carrus $^{\mathrm{b}, *}$, Ricardo Garcia-Mira ${ }^{\mathrm{a}}$, Fridanna Maricchiolo ${ }^{\mathrm{b}}$ \\ a University of A Coruña, Department of Psychology, Spain \\ b Università degli Studi Roma Tre, Dipartimento di Scienze della Formazione, Italy \\ ' Sapienza Università di Roma, Dipartimento di Psicologia dei Processi di Sviluppo e Socializzazione E' CIRPA-Centro Interuniversitario di Ricerca in \\ Psicologia Ambientale, Italy \\ d University of Genova, Anthropological Sciences Department, Italy
}

\section{A R T I C L E I N F O}

\section{Article history:}

Received 3 April 2015

Received in revised form 20 October 2015

Accepted 9 December 2015

Available online $\mathrm{xxx}$

\section{Keyword:}

Energy consumption

Organizational sustainability

Workplace behaviors

Qualitative research

\begin{abstract}
A B S T R A C T
Large organizations are responsible for a significant amount of GHG emissions. This trend will even increase over the next 100 years. An issue for environmental research is the investigation of the factors promoting or hindering the transition to more sustainable energy behaviors in the workplace. This study is part of a larger project, funded under the EU-FP7 program, called "Low Carbon at Work" (LOCAW). We present the results of a qualitative study assessing the existing everyday behaviors in two large-scale organizations: an Italian-based multinational renewable energy producer, and a Spanish public university (The University of A Coruña). Data obtained by interviews with key-informers and focus groups were content analyzed, using ATLAS.ti. Results indicate many commonalities and some differences between the two case studies. Working in a green anergy company leads individuals to be more sensitized about environmental issues, although a core thematic category refers to the concept that energy-related behaviors "rely on individual feeling". Data from the University of A Coruña suggest this organization has the right values and objectives, but it does not always adequately implement organizational policies to support sustainable energy use among students and employees. Theoretical and practical implications are discussed.
\end{abstract}

(C) 2015 Elsevier Ltd. All rights reserved.

\section{Introduction}

Despite the overwhelming scientific evidence, climate change remains a contentious and contested issue, so that many individuals and countries display skepticism on the anthropogenic causes of global warming (e.g., [67]). In present times, reducing carbon emissions is a fundamental goal for the transition towards more sustainable human societies, and it is reflected in official goals and political strategies of supranational authorities and intergovernmental bodies at the global level. As an example, we could mention here the 2009 Review of EU Sustainable Development strategy, which underlines how the EU has taken the lead in the fight against climate change and the promotion of a low-carbon economy (http://ec.europa.eu/environment/eussd/), although the

* Corresponding author at: Roma Tre University, Department of Education, Via Milazzo 11B, Rome 00185, Italy. Fax: +39 0644703879.

E-mail addresses: giuseppe.carrus@uniroma3.it, carrus.giuseppe@gmail.com (G. Carrus). efficacy of traditional political responses to energy-related issues is still a matter of academic debate (e.g., [9]).

In this broader context, energy-related issues have acquired increasing centrality in the current agenda of social science research (e.g., [24,31,59]). The concept of energy transition [58], and its socio-cultural implications (e.g., [53]), has also been underlined as crucial to explain societal changes of this kind (e.g., [23]), and is particularly interesting in the domain of low carbon energy policies (e.g., $[2,7,20,33,41])$. Large organizations are an important player in this transition, as they are responsible for a significant amount of GHG emissions. Estimates for the year 2000 of the Emission Database for Global Atmospheric Research, from the Joint Research Centre of the European Commission ( http://edgar.jrc.ec. europa.eu/), which considered 8 different categories of sources of GHG emissions (industrial processes, power stations, transportation fuels, among others), suggest that the potential contribution of large organizations to global warming over the next 100 years will be highly significant. Understanding the key factors affecting sustainable changes in everyday behvaiors in the workplace is then 
a worhty issue for social science research, in view of a transition towards more sustainable societies.

\section{The challenge of sustainable organizations}

Redressing the ongoing and preventing the future ecological degradation is one of the most prominent challenges organizations face today [68]. Organizations and their employees are one of the largest users of the worldís energy resources [35,45,56,62,63]. Demands for social and environmental responsibility in organizations, together with raising consumption of energy and associated costs have led to an increase of the interest of organizations in ways to promote energy conservation at work (see for example: $[1,54,60])$. To date, however, most common strategies have focused on structural and operational changes such as removing or changing inefficient equipment or changing business processes, as these changes tend to be easy [54]. Even where they can have a clear impact, the environmental effectiveness of technological solutions to reduce carbon emissions is contingent upon the way in which users engage with and deploy them [40]. Previous research has also indicated that, besides structural and technological changes in organizations, substantial reductions can be achieved even by behavioral measures alone [19].

Most of the existing research focusing on sustainability in organizations has looked at their performance at an aggregate level [38]. However, much less research has focused on the environmental performance of organizations in terms of their attempts to encourage employees to behave pro-environmentally or to come up with initiatives and ideas to change organizational practices, processes or products in the direction of sustainability. This has been in spite of the evidence that a good level of pro-environmental performance of an organization might make it more attractive to competent prospective employees $[28,29,65]$. Labor productivity might be higher, as it has been suggested that individuals who choose to work for "greener firms" might work harder [6]. Recent research has also suggested that the adoption of environmental standards, and the organizational changes they entail, might result in higher labor productivity and improved interpersonal contacts within organizations (e.g., [18]). Different mechanisms can be identified at the basis of this effect: first, working for a more environmentally friendly organization might boost employee's identification with their company and this, in turn, is reflected in higher productivity. Second, implementation of employee training programs in the environmental domain might lead to higher levels of interpersonal contacts and employee engagement; this, in turn, helps the process of knowledge transfer and the generation of innovative ideas, promotes job satisfaction and motivation, and finally leads to increased productivity [18].

It shall be noted that while individual pro-environmental behaviors are undertaken entirely voluntarily as part of oneís personal life, employee green behaviors always involve some degree of organizational oversight [44]. Individual contributions to organizational sustainability goals can be quantified by rating the frequency and proficiency with which employees engage in pro-environmental behaviors. They can be undertaken as part of the tasks that the employees are required to carry out, or outside the formal duties and responsibilities in work contexts [44].

An interesting contribution on these issues comes from a recent work by Paillé and Mejía-Morelos [46]. These authors start from the consideration of the unconsistent results of studies on pro-environmental behavior at work that employed traditional theoretical approaches developed in environmental and social psychology, such as the value-belief-norm theory (e.g., [55]), the cognitive theory of stress [32], and the theory of planned behavior [27]. While, these theories have been useful to explain pro-environmental behavior in household settings, new theories and frameworks are needed to explain employeesí behaviors on the job that would take into account the specificity of these settings. To this end Paillé and Mejía-Morelos [46] propose the social exchange theory (SET) as a suitable framework for the understanding of sustainable behaviors in organizations (see also Ref. [16]. A basic assumption of SET suggests that individuals are driven by the outcomes they expect and actually get from relevant others (e.g., [69]). Therefore, the authors argue that employees might be more willing to undertake sustainable behaviors at work when they perceive that the organization is actively engaged in initiating, developing and maintaining favorable work conditions. Consistent with this idea, these authors provided empirical evidence that organizational support positively affects the pro-environmental behaviors of employees (via positive job attitudes), while a breach in the psychological contract between the employees and the organization would undermine this positive path [46].

Among the specific organizational factors that have been proposed to have an effect on pro-environmental behavior in organizations we can mention aspects such as organizational focus, organizational structure, organizational/site type and size, departmental type and size, and organizational culture [64]. In particular, organizational focus and culture have been proposed as the most straightforward determinants of pro-environmental behavior [37]. Characteristics of leadership and leader's behavior are also important factors for the transition to more sustainable organizations (e.g., [4,49]). Leaders influence employee behavior through the setting up of descriptive norms that either support or hinder proenvironmental behavior. Descriptive norms have been defined as individual perceptions of what most other people do, and they have been shown to play a relevant role in human behavior by conveying social information about what is a normal or appropriate behavior in a given situation or context (e.g., [15]). Their effect has been studied for a wide range of human behaviors, and their link to pro-environmental behavior has been repeatedly confirmed in empirical research (e.g., [13,30]). Leadersí beliefs and actions play then a particularly important role in the perception of what constitutes an appropriate behavior in the workplace [50]. Likewise, recent evidence shows that perceived co-worker's support might encourage eco-helping among colleagues and engagement in proenvironmental behavior at work [47].

\subsection{Energy-related behaviors in the workplace}

As a fundamental domain of human everyday life, work is a place and space where the sometimes contradictory demands of economic profit and environmental sustainability meet and are negotiated, with the resulting effects on individual behaviors, energy consumption and greenhouse gas emissions. As people spend an important part of their lives at work, within a community of values, norms and everyday actions, it is also the place where identities are defined, where individual values are transformed and where sustainability-related behavior can be either promoted and rewarded or hindered and discouraged [10]. Nevertheless, research on sustainable everyday behaviors at work, and on the factors promoting or hindering them, is still not abundant within social and behavioral sciences and environmental psychology (e.g., [61]). Specifically, a recent systematic review of the literature has argued that, among other gaps, there is insufficient evidence on the role of social norms on pro-environmental behavior in organizations, including the role of a lack of pro-environmental social norms; it has been also pointed out that there is little research available on general organizational determinants such as organizational structure or culture and their effects on specific behaviors; and that more qualitative research is needed to uncover the relationships between organizational and individual factors in organizations [38]. 
Although household research has shed light on the determinants of pro-environmental behavior, it has been noted that translating findings to a workplace context is not easy due to two main reasons: first, the costs of consumption are different in the household and in a work context [57], and secondly, organizations' size, structure, goals, culture and other organizational factors might also produce different conditions for pro-environmental behavior than those present in households [38]. For example, studies focusing on energy behaviors in organizations have shown that substantial energy savings targeted by an organization may be limited by wasteful employee behaviors [58], so that employees can have a significant contribution to energy use in organizations, both directly and indirectly [25]. A recent qualitative study by Lo et al. [37] looked at four organizations (two private companies, an NGO and an university), which shared some form of commitment to environmental sustainability and did not have energy conservation as their primary aim. This study identified a series of important barriers to energy conservation behaviors of employees in the workplace, such as the lack of organizational communication on environmental issues or the lack of adequate information on what are the behaviors to perform, and their relevance in terms of environmental impact. The findings of this study suggest the importance of focusing on the workers' perceptions of barriers to and drivers of pro-environmental behavior in the workplace, as a way to design more environmentally effective organizational contexts.

\section{The present study}

The study reported here is part of a larger project on everyday sustainable behaviors in 6 large scale organizations across Europe. The project, called "Low Carbon at Work" (LOCAW), was funded under the 7th Framework Programme of the European Union. The projectís aim was to provide a comprehensive perspective on the barriers to and drivers of sustainable behaviors in organizations, and to formulate and test a series of policy recommendations in this domain. Within the LOCAW project, three main categories of workplace behaviors were investigated: (1) consumption of materials and energy; (2) waste generation and management; (3) organization-related mobility. While the main research questions of this project focused on how the organization as a whole could become more sustainable, in this paper we undertake specifically the question of what are the main barriers and drivers for organizations to becoming environments that provide motivations and space for employees to carry out pro-environmental behaviors. On the one hand, we were interested in understanding the factors affecting energy use in work-related behavior, and, on the other, our goal was to understand those conditions that might contribute, either positively or negatively, to the active engagement of workers in providing suggestions for alternative ways of carrying out tasks or improving infrastructure that would ultimately lead to energyuse reductions, which constitutes a more engaged and pro-active form of pro-environmental behavior.

Recently, it has been argued that the state of the art in the literature regarding the promotion of pro-environmental behavior in the workplace does not allow the formulation of a comprehensive framework on organizational determinants that directly influence pro-environmental behavior at work. Also, interorganizational analysis of the relationship between organizational and individual determinants of pro-environmental behavior at work was deemed inexistent [37]. In order to understand the relationship between organizational and individual factors, and the patterns of barriers and drivers generated by their interaction, the present study targeted employees from two different types of organizations: a private green energy company and a public university.
To this purpose, a multi-method qualitative approach was used to identify: (i) the main organizational and individual factors affecting pro-environmental behavior in the workplace; (ii) the resulting barriers to and drivers of pro-environmental behavior that result from their interaction. Given the lack of clear empirical evidence on the interactions between organizational and individual determinants, and the resulting barriers and drivers in this domain, a qualitative methodology was deemed as more appropriate, by means of in-depth interviews and focus groups.

\subsection{The case study organizations}

Two different case study organizations were chosen, in two different European countries: Italy, and Spain. Although cultural differences between these two western European Mediterranean countries might be a relevant issue for research in this field, our focus here is more on the differences between the two specific organizations under study. Both of them were in fact considered as particularly suitable to our purposes.

In the case of Italy, we focused on a renewable energy provider, which operates in the global market as a key player having energy as its core business, while in the case of Spain we focused on a public university. Clearly, renewable energy companies are a direct stakeholder in the transition towards more sustainable energy production and consumption. Therefore, they represent an ideal arena to investigate how the environmental and energy-related practices of the employees (and citizens in a larger sense) are framed and shaped by organizational factors, and by the broader social context. Likewise, universities are also key actors in sustainability transitions as both workplaces and learning communities. Their direct and indirect impact on current society is considerable in terms of training citizens who are knowledgeable of environmental problems, know how to act in sustainable ways both in their homes and at their workplaces, and are consistently motivated to do so. Thus the two organizations we chose are both strategic actors in driving sustainability transitions, and both have a key educational role in the sustainable behavior of employees and citizens.

The two organizations also have interesting differences from the perspective of the objectives of this study. The Italian green energy provider is a private company operating in a competitive global context, while competitive pressures and market constraints are less affecting the Spanish university as a public organization. An elected management team democratically governs the latter, which is not the case of the private company, who operates according to the standard business corporate procedures in a market context. This aspect is likely to generate different opportunities and constraints for both leaders and general workers in each organization.

\subsubsection{An Italian renewable energy company}

The organization chosen as the Italian case study was a major green energy producing company, with about 3,600 employees. Founded in 2008, this company is dedicated to developing and managing energy generation from renewable sources at an international level. In the European context, this company operates in Italy (where it is particularly active in supplying energy from three renewable energy technologies: geothermal, hydroelectric and solar), as well as in Spain, France and Greece. Outside Europe, it operates in the United States Canada, Central and South America. Only in 2013, thanks to renewable energy generation, this company contributed to save over 16 million tons of $\mathrm{CO}_{2}$. Beside its main mission, which is intrinsically committed to carbon emissions reduction, the company has started since 2013 a plan that aims at putting the sustainability concept at the core of corporate procedures, which includes short-, mid- and long-term actions aiming integrating sustainability into the various phases of the company's value chain. For example, the 2013 consolidated financial 
statement included, for the first time, several environmental, social and governance indicators, as a first step for the integration of nonstrictly economic and financial results in the company periodical business reports.

\subsubsection{A Spanish public university}

The chosen university is a public organization situated in the province of Galicia and founded in 1989. It has two campuses (with 8 spatial locations), for a total of 1,513 faculty and 760 administrative and service personnel. It has 24,554 students. The university has established an action strategy for sustainability at the institutional level, and it has specified it in a general strategy and a series of sectorial plans for each domain. It has also created the Office for the Environment, which is in charge of promoting and implementing measures and campaigns to reduce the greenhouse gas emissions of the university and to promote pro-environmental behavior among both workers and students. The Sustainability Report published each year by this office shows that direct energy consumption accounts for the $38 \%$ of the university emissions. If we consider the indirect energy consumption embedded in mobility, for example, this percentage is even much higher. Achieving reductions in energy consumption is thus declared as a priority by the organization, as part of its emissions reductions goals.

\subsection{Recruitment and participants characteristics}

The organizations were contacted with an invitation to participate in the study and were promised a final report with recommendations for strategies to promote the pro-environmental behavior of employees. After obtaining the agreement of participation from the organizations' leaders and top managers, we used both document analysis and preliminary meetings with a few key informants to identify other relevant key informants and all members in key leadership positions.

Different sources of data were considered. As a preliminary step, we undertook an analysis of relevant organizational documents (e.g., websites, brochures, promotional and advertising flyers, organization charts, environmental and social reports, codes of ethics etc.), in order to get a clear picture of the explicit environmental commitments manifested by each organization, as well as the extent to which organizational values are implemented in both internal and external documents. After this initial phase of desktop research, we carried out a series of interviews with key informants, in order to understand the perceptions of workers about the importance given to environmental behvaiors in the organization, and their informed understanding of barriers and drivers to sustainable energy use. Finally, a series of focus groups were carried out, to obtain a richer perspective emerging from the spontaneous interactions among members of the organizations.

A total of 19 in-depth interviews were carried out with key informants in each organization (10 for the green energy-producing company and 9 for the public university). Key informants were high-level management staff in relevant positions from a sustainability perspective, purposively identified for having different work roles and responsibilities (e.g., for the energy company: Industrial relations, Safety, Planning, Operation and maintainance, Energy management, Engineering, Administration, Sales, ICT; for the university: Infrastructure, Economic Planning, Office for the Environment, the Social Council). In the universityís case, representation of both university and department level of management was also ensured, as well as representation of both academic and administrative staff, and representation of the two main campuses.

Three focus groups were carried out (two for the university and one for the green energy company) and the number of participants for each focus group ranged between 6 and 10 participants. Participants were not offered any payment or incentive for their participation. In the case of the green energy company, 10 persons took part to the focus group. As in the case of the interviews with key informants, the composition of the group was defined in accordance with the company management, after preliminary operative meetings. Participants were recruited through a first invitation from the company management, and then received a further brief explanation of the objectives of the project by the research team prior to the participation. Participants differed for their relative work experience field of activity, and seniority of service in the company. Six participants were men and 4 women. Nobody declared to be member of any particular environmentalist association, neither member of a specific trade union. In the case of the university, in order to select a suitable sample for the objectives of the focus groups, an initial list was compiled that contained all persons that occupied positions of responsibility related to the three behavioral domains focused by the study. Both staff in political (elected) and non-political (administrative) positions were listed (resulting in a number of 20 members of staff). Besides staff, a number of students that held representative positions on both campuses were identified and invited to participate in the study. A number of 14 members of both staff and students accepted the invitation to participate, 8 for the bigger campus, and 6 for the smaller one. The focus groups included senior members of the university with responsibility over infrastructure and maintenance decisions, environmental and economic policy, and general budgetary and oversight functions. They also included a few key union representatives. Both had a relatively good gender distribution: the larger focus group had a composition of 3 female participants and 5 male, while the smaller focus group achieved an equal distribution of three female and three male participants.

\subsection{Interview and focus group guides}

A guide was designed for each data collection source, which was used as a track to conduct the individual interviews and group discussions, respectively. Sufficient flexibility was allowed for the researcher to pursue themes that arose spontaneously and were informative for the objectives of the study. The in-depth interview guide addressed more on the role of structural and organizational factors influencing pro-environmental behavior at work, while the focus groups addressed more on the specific barriers and drivers of behavior that were generated by the interaction of organizational and individual factors.

\subsection{Data analysis}

Both interviews and focus groups were audio-recorded, after getting the informed consent of the participants, and lasted between 35 and $90 \mathrm{~min}$. All the material analyzed was stored in electronic format, and was subjected to thematic content analysis procedures, using ATLAS.ti [5,52]. Thematic analysis is a conventional practice in qualitative research, which involves searching through data to identify recurrent patterns. A "theme" is a cluster of linked categories conveying similar meanings and usually emerges through the inductive analytic process that characterises the qualitative paradigm. Like in classical grounded theory approaches (e.g., [26]), the exploratory power of this technique can be enhanced when the analyst is not guided by strong preconceptions in the analisys. Thus, the analyst does not necessarily have to be an expert in the research topic, although a researcher must have at least some conceptual understanding guiding the insight processes; usually, the literature review is only delayed rather than totally skipped (see also Ref. [14]). Since its proposal at the end of the sixties, in the very early days of the (re) emergence of qualitative methods in social sciences, there have been different approaches to and versions of grounded theory, with different theoretical underpinnings 
and procedures (for a useful overview, see Ref. [3]). However, the idea of not fully engaging with the relevant literature prior to beginning the data analysis, to avoid it being shaped by preconceptions from existing research rather than being truly grounded in the data, represents a basic assumption in this approach. The distinction between "hard" and "light" grounded theory approaches might be useful to better describe the method we followed in our study [48]. A hard grounded theory approach requires the implementation of the full range of grounded theory procedures, including theoretical sampling, with the aim of producing a theory grounded in data. Here is one notable difference from thematic analysis: although thematic analysis can produce conceptually-informed interpretations of data, it does not attempt to develop a theory. A hard grounded theory is rarely used, even when a grounded theory method is claimed, and is usually achievable only in large-scale dedicated research projects [48].

A light grounded theory approach involves using the techniques of grounded theory for the development of categories (and concepts), and an understanding of the relationship between the various categories and concepts [48]. This is probably the most frequently used form of grounded theory. The practice of thematic analysis is similar to light grounded theory, although differences in terminology-e.g., categories vs. themes-can mask the underlying similarities. Both involve coding and the generation and interpretation of broader patterns in data: the core concept in grounded theory is "code", which is a common label in many approaches to qualitative analysis.

The ATLAS.ti software allows the selection of units of meaning (so-called "quotations") directly from the audio archives, and these can be assigned specific codes, which capture the theme of each quotation. Each code will in the end have several quotations assigned to it, thus allowing the researchers to identify the most commonly emerging themes. Codes can be connected among themselves through logical relationships; families of codes can be constructed for each superordinate category of meaning, thus providing a map of themes and relationships emerging from the analyzed material. Each code had two numerical indicators associated to it: the number of quotations assigned to a particular code ("Grounded"); and the number of relationships a code has with other codes ("Density"). In practical terms, codes that have a high frequency and/or a high density reflect the most important and central themes in the analyzed material.

\section{Results}

It is beyond the scope of this paper to illustrate the details of the specific Atlas.ti findings at the micro level for each single case study under investigation. Rather, our purpose here is to provide an overarching narrative about the comparison of the overall trends emerged across the two different organizations considered. Therefore, for the purposes of simplicity and clarity, and also to facilitate the comparison between the main trends emerged in the two case studies, we will follow a discursive format in the presentation of the results, jointly for the interviews and focus groups materials.

\subsection{Organizational background: preliminary document analysis}

The document analysis carried out in the two organizations reflects more similarities than differences between the two. Both organizations express a strong formal commitment with sustainability in general, and with energy conservation in particular. They present specific environmental objectives and make reference to specific strategies for their implementation. Also, both organizations describe environmental objectives as being value-ladden aims that would need to be pursued in all circumstances. How- ever, while in the Italian company, energy reduction objectives are considered part of the values the whole company endorses, for the Spanish university, the documents emphasize the role of the management teamís sensitivity and endorsement of pro-environmental values. This difference already points out to a different type of motivation that is present in each of the two organizations: for the Italian company the external market context in which they operate generates a driver for pursuing pro-environmental objectives; in the case of the university we have an internal democratic context (in which leadership is elected for office) performing the same function. Furthermore, both organizations emphasize the driver role of external political regulations at both European and national levels, in generating incentives for organizations to implement strategic policies that lead to emissions reductions. Nevertheless, the relative instability of national incentive policies is seen as a potential barrier to sustainability transitions in the case of the Italian energy company.

\subsection{Organizational barriers and drivers to pro-environmental behavior}

In the in-depth interviews, a special emphasis was given to the structural and organizational factors that might generate specific barriers and drivers to pro-environmental behavior in general, and energy reduction in particular. However, the interview format was open enough to allow for the emergence of all themes that were relevant for energy-related behaviors, including individual ones. Focus groups went a step further and addressed on the interactions between organizational and individual factors and the resulting patterns of barriers and drivers to pro-environmental behaviors and sustainable energy use in the workplace.

\subsubsection{Pro-environmental and energy behaviors in the two organizations}

We inquired about behaviors such as temperature regulation, light switching, and behaviors related to the use of printers and other electronic devices, such as the frequency of printing and placing computers and printers in standby, among others. Our results show that organizations have been successful in promoting low-cost pro-environmental behaviors, such as reducing printing frequency or placing computers in standby. These have become habitual behaviors in the case of the university, for example. However, temperature regulation is not possible, as its control is automated and assumed by the university and considered to be inefficient by workers, both in terms of comfort and environmental impact. Light switching when leaving the room is also a problematic area of behavior, as some interviewees point out that leaving the lights on is sometimes done as a way of signaling presence at work to superiors. This indicates an element of organizational culture that focuses on control of the physical presence of workers, that acts as a barrier to sustainable behavior.

In terms of the transformations of infrastructure leading to higher energy efficiency, organizational documents revealed that the university has undertaken a few such projects, installing, for example, renewable energy sources for several heavy consumer buildings. It is thus striking that these measures do not seem to be known by regular workers (with the exception of a few members of the management team that were involved in their approval), which indicates that pro-environmental policy is not well communicated in this organization. Our data suggest that this might be due to two factors. First, infrastructure change tends to be done in a top down fashion and delegated to the Environmental Office, which carries out the projects; second, the channels established for the communication of environmental policy consist of reports and news on the website of the Office, with some presence in specifically established social networks. Exploring more creative 
options to communicate environmental policy would then potentially improve employeesíawareness of organizational efforts to promote energy efficiency, a key element of organizational climate [42]. The two excerpts reported below are good examples of this issue.

Participant 1 (University): "The only information I receive is through email and occasionally the Office does a survey and they ask how much paper we spend and stuff like that, and it is a pity, a lost opportunity, they could provide us with more recommendations on what types of paper we should use, or how we could save energy".

Participant 2 (University): "There is no policy on energy saving, for example, and no information on what should be done. It seems there is a communication problem and they could give us suggestions and recommendations, good practices, things like that. There is none of that. And when as a Unit we have to make decisions we say, letís see, this company could be my provider for paper and we do not think about other things, there is inertia, and if the company works well we do not change it and do not take environmental issues into account."

\subsubsection{Perceptions of the role of external context}

Employees in both organizations emphasize the important role played by the external context, which is perceived mostly as generating barriers to the promotion of pro-environmental behavior in the workplace. Two important barriers are emphasized by both organizations: infrastructure-related barriers, and cultural or normative barriers.

Infrastructure-related barriers refer to the adaptation of buildings and efficient public transport systems (mobility-related behaviors were also investigated, because of their relevance for indirect energy consumption). Inadequate infrastructure is considered a cause for unsustainable energy behaviors, and in both organizations it seems that costs are a deterrent for massive infrastructure improvement. In the case of the green energy company, such kind of barrier is also directly linked to the inefficiency of the urban transportation system. In fact, the company headquarter is located in the center of Rome, where low carbon urban mobility is particularly problematic because of the poor public transportation network and the resulting huge car traffic congestion. This makes it difficult to fully implement the specific low carbon mobility policies that the company is pursuing:

Participant 11 (Green energy company): "Mobility issues are different according to the place where you live. We live in Rome and we experience many problems. Notwithstanding our company policies, we have problems because we live in Rome. I see many colleagues of mine, they live in other cities...then, it is not worth to intervene with particular solutions".

Participant 12 (Green energy company): "However, we are in the city centre and it does not help. Firstly, the headquarter's street is not well connected. It is true that there is the metro nearby but when you need to arrive by car you have the problem of parking. I need to arrive by car because I take my children to school and I come here. Then, about mobility there are many things to do; it is necessary to rethink geographical aspects".

There are however two important trends that we could detect in both organizations: first, environmental energy policy is often equated with infrastructure change, which has high initial costs, while emissions reductions that could be achieved through behavioral change policies are either not mentioned at all or downplayed. This seems to be a belief held by members of the management teams in both organizations, which provides an explanation for the lack of behavioral change strategies, that can be observed in both organizations (although there are some attempts in both organizations, but not specifically related to energy issues).

Participant 10 (Green energy company): “Looking at resources consumption, I guess there are cultural obstacles. I mean, in our company many people have a different cultural background [from mine], maybe because they are younger. They pay a greatest attention to these problems because we are bombarded with message about attention to be due to resources consumption.

Participant 4 (University): 'My generation did not receive this type of (environmental) education and thus I must say it still entails an effort for me to separate waste, for example, in my house, because it is something I acquired as a habit recently. And also I should say, there is no environmental culture in this country, not like in others, but it is also true that in others policies started earlier'.

Cultural and normative barriers in the wider context refer to the support that pro-environmental behaviors have in society at large according to the interviewees, and in the direct social and political environment in which the organizations operate. In the case of the university, the larger cultural context is perceived as unsupportive to environmental policies and norms. This characteristic leads to environmental objectives being perceived as value-ladden objectives, to be undertaken when budgets are considerable and passed to the background when they are limited. The economic crisis is thus mentioned often as a barrier to efficient energy policy, especially when conceptualized as infrastructure adaptation and radical technological change. This perception influences motivation to perform pro-environmental behaviors, especially when these are costly.

Participant 3 (University): "Letís see, I think that the problem is cultural in our society, there is no energy saving, and it is also tiring to go after people and turn lights off or find water left running in the bathrooms. There is no awareness energy has a cost, as here nobody pays for it directly. And the same happens for heating, no concern for energy efficiency in this building, it consumes a lot of diesel. And this has to do with how it was built, all these new modern buildings, which are supposed to be beautiful, they do not have good ventilation systems and were not built with environmental criteria in mind.“

Participant 5 (University): "Now, we took other measures and we do not have a clear idea whether it is economically profitable but we think environmentally they are positive so this government has implemented them anyway. And we would have liked to implement more, but economically it is imposible and it is inevitable that we need to take economic aspects into account but if we looked at that, it would be imposible to take any environmental measures so one has to do that by ignoring them, if you can afford that of course."

Our findings also reveal a tendency to attribute responsibility to wider contextual factors and downplay the impact that organizational efforts could have on promoting pro-environmental behavior both for their employees and in the wider societal context. It seems that both organizations shy away from their role as potential frontrunners in transitions to sustainable energy behaviors, and this is also reflected in the intervieweesíperception that although both organizations have a formally expressed commitment to sustainability, they do not make sufficient efforts to promote proenvironmental behavior at work.

Participant 4(University): "I think on the one hand there is a lack of sensibility and I donít think politically it is very profitable and governments do not have that mentality, so...this is how it is. 
At the same time, in the case of the green energy company, it also emerges the awareness that the promoting a more efficient energy use in the workplace does not necessarily require massive structural change; rather it is still a mostly cultural issue:

Participant 14 (Green energy company): 'There are not obstacles, just cultural factors, such an organizational laziness, a cultural laziness. If our C.E.O. would decide to change our work habits, organizational lifestyle I mean, there are not severe obstacles'.

\subsubsection{Organizational tensions between expressed commitment and actual behaviors}

In the analysis of both organizations we find contradictory themes that refer to, on the one hand, the strong organizational commitment to pro-environmental values, and the perception that the organization is not doing enough on the other hand. While commitment is perceived as a driver for pro-environmental policies, the causes attributed to the lack of organizational strategies for sustainability range from high costs, to inefficient internal structures for the communication of environmental policy, to insufficient consideration for employeesísuggestions for improvement to reach the adequate levels of decision-making. The fact that high costs are perceived as a barrier shows that environmental goals are considered secondary to economic considerations in both cases. The following excepts are a good example in this sense:

Participant 3 (University): 'There is a will to take environmental criteria into account but also there is an economic criterion. We try to do something and harm the environment as little as posible but of course only if it is not a lot more expensive.'

Participant 6 (University): "If we consider the environment as an afterthought then when we need to reduce spending we cut, barbaric as it is, the nonessential things become education, culture, social responsibility, environmental education, and we become preocuppied with reaching the end of the month and when times are better we go back again. This is a serious mistake, and this is definitely not social responsibility."

While, in the case of the university, economic restrictions are determined by the assignment of public budgets, this finding becomes more interesting in the case of the green energy company, whose core business is indeed renewable energy production. This apparently paradoxical circumstance might be related to a sort of compensation effect, by which the organization already sees itself as intrinsically contributing to a sustainable transition goal, and thus not so much "forced" to be concerned with the performance of its employees in terms of environmental impact, despite the increasing evidence about the positive links between environmental and economic corporate performances (e.g., [51]; see also Ref. [11]). In the case of the green energy company, these contradictions emerge clearly from our data. In some cases it emerges an apparent contrast between the organizational mission and its formal commitments, and the actual situation of the workplace setting.

Participant 13 (Green energy company): “As for energy consumption, there is not a great consistency between our external communication, about the attention to be payed to energy saving as sustainability factor, and our company. If you consider this building from the point of view of the energetic profile, it is severely inadequate".

A significant difference can be found in terms of the internal communication of pro-environmental policy in the two organizations. At the university this is considered to be one of the main barriers to pro-environmental behavior, due to the fact that this lack of communication generates a perception that the organization is not really committed and doing enough to promote pro-environmental behavior. Conversely, in the green energy company the internal structures of communication seem to work well as a driver for acting pro-environmentally. Interviewees reflect on the fact that being in a company whose organizational focus is on sustainability has contributed to both significant learning in terms of appropriate green behaviors, and also to transferring some of those behaviors from the work to the home domain. Also, the organization has set structures in place to promote the creative formulation of suggestions from employees, on how to innovate its energy-conservation practices, through the set-up of a competition, which is incentivized by the company. Besides showing organizational commitment to energy conservation, such structures also create some possibilities for employees to provide suggestions for sustainable change, and thus potentially take a more active role in the formulation of organizational policies.

Participant 10 (Green energy company): "Working in this company changed a lot of behaviors; it fostered our sensitiviness towards energy saving. I have to say that at home I take great care that my children turn off lights, for example. I am very accurate to educate them to behave responsibly when they use resources, such as energy or water.

Participant 11 (Green energy company): 'Sincerely, I have to say that the impulse for people to separate waste helps to increase our sensitiviness. In this way, you feel it becomes a daily habit. Here, we are close to this issues'.

However, the existence of such a policy does not automatically lead to generalized and stronger pro-environmental behaviors of employees. Pro-environmental policies are not sufficient alone, and need to be complemented with a clearer exemplary behavior by the organization itself. The spreading of organizational pro-environmental norms would more likely support the proenvironmental behavior of the the employees. Previous research indeed indicates the role of organizational focus in workplace proenvironmental behavior [64], and has signaled the lack of research on the role of organizational norms, including environmental ones [38]. This aspect will be discussed in the next paragraph.

\subsubsection{The role of organizational norms as drivers of pro-environmental behaviors}

In general, employees of both organizations do not refer often to the role of social influence processes, although several identified themes point to the role of norms in shaping pro-environmental behavior in the workplace. For example, in the green energy company, interviewees affirm that informal rules work better than sanctions and prescriptions. The psychological mechanism at the basis of this process relies on the importance of adopting a 'promotion' focus for channeling individual positive behaviors in the direction of sustainability, rather than a "prevention" focus, which would rely on the sanctioning of undesired behaviors (e.g., [8]). Indeed, the promotion of responsibility toward energy sustainability might operate as a true and proper behavioral driver, by nurturing individual motivation for pro-environmental behavior. On the contrary, sanctions and bans often work as a barrier restraining proactivity and initiative, particularly in the context of a large scale organization.

In the case of the university, making organizational efforts to promote pro-environmental behavior more visible could support a shared view of the university as an institution that gives importance to sustainability issues, and in turn contribute to enforce a culture of sustainability [43]. Organizational leadership and academic staff 
undertaking on pro-environmental behavior can become relevant examples for workers and students alike.

Organizational culture is also reflected in the social norms present in the organization, signaled by the behavior of both leaders and peers. Our results in this case suggest that the behavior of leaders is not always seen as reflecting a commitment with environmental objectives. This issue deserve further inquiry, as previous research show that the exemplary actions of leaders, leadership support of employeesí pro-environmental behavior, and descriptive and injunctive pro-environmental norms are important determinants of behavior $[4,42,50]$.

Participant 6 (University): "Is there a social demand for environmental issues? I think it exists and it is growing. Corporate social responsibility is the biggest development of the 21 st century. In public organizations, there was at the beginning the belief that they do not have to apply them as they are already dedicated to public service. Today, all that is left behind and we all assume that all organizations have to assume social responsibility. Social sensitivity exists but to what degree do universities feel it? In all organizations, worker environmental awareness, concern and implementation of measures are proportional to the awareness and concern of their leaders. So the government team has to really endorse environmental values."

Participant 13 (Green energy company): "There is not any doubt that this company pay great attention to energy saving. Generally speaking, it is an organizational mission because there is such an 'ethic soul' in environmental issues. It follows an increasing attention to keep low levels of energy consumption and saving behaviors".

\subsection{Individual barriers and drivers}

\subsubsection{The role of attitudes}

The most important attitudes we identified as relevant determinants of pro-environmental behavior are related to the causes and attributions of responsibility when referring to pro-environmental behavior, as well as the distribution of responsibility between the organization and the individual. In both organizations, interviewees consider the pay-off of performing energy-reduction actions as an important barrier affecting sustainability in the workplace. That is, our respondents consider that energy behavior in the workplace is less sustainable than behavior at home, because unsustainable behavior at work has less direct financial consequences for the individual.

Participant 5 (University): "The fact that nothing costs is an issue. . I never liked wasting paper, and I turn off the light when I go out of my office, and it depends on the person, I think this is more a personal issue, and as the person responsible for energy systems, I have this awareness. But there are people that ask you for a new radiator to reach a temperature of 25 degrees Celsius and I try to explain to them that every degree means this much more consumption, but. . .it is not easy. And in general, I would say there isnit much awareness. At home energy has a cost for people, but not at the university.“

Participant 3 (University): 'Energy does have a cost, as the university bill for energy comes directly out of our taxes. A public university draws from out common pocket as well. Again I must say, that we lack environmental education but that does not mean that socially, as a group, there isnít any change, especially in the younger generations, and also, it is true that, but this is an issue in this country in general and not only of this university, and there is a general belief that what belongs to everybody does not belong to anyone.'
Furthermore, interviewees refer to the interaction between the organizational context and the individual free-will. For example, in the case of both the university and the energy company, participants acknowledge that existing good practices are a result of the interaction between top-down conditions and individual behavior, but that sustainable choices finally rely on individual 'feelings'. However, responsibility for change is assigned more directly to the university as an institution, or the company as a whole body, and not only to individual members. We identified more thematic codes referring to what the organization could do, rather than to what the individuals within it could change in their behavior. Participants also refer to sustainability as a highly complex issue within the organization, which require "virtuous" interactions between structural and individual elements.

\subsubsection{The role of values}

Although a lot of the value-related content we identified refers to the conflicts of values at an organizational level, as mentioned before, employees also acknowledge the role of individual pro-environmental values, education and awareness as important determinants of positive energy behaviors. In the case of the focus groups carried out at the University, for example, the theme of the "Importance of environmental education and awareness" has the highest numbers of quotations associated. Thus, we found that although employees attribute the highest responsibility for the promotion of pro-environmental behavior to the organization, they also consider individual determinants as relevant for the success of organizational transitions to sustainability. This feeling of individual responsibility can constitute a driver for compliance with organizational policy, when this is perceived as based on positive stimuli for behavior and not sanctions. It can also constitute a basis for pro-active engagement in finding innovative solutions to improve energy efficiency in the workplace.

These references to individual determinants of behavior suggest that organizations could do more to promote contexts in which workers can autonomously carry out their pro-environmental choices and become actively involved in shaping organizational efforts to sustainability. This would probably lead to increased perceptions of energy efficiency as a partnership between the organization and the employees to achieve a higher-order sustainability goal.

Participant 2 (University): "I think what is missing is active policies, someody who is concerned about this and tries to do something. Starting from the leadership, if they try to do something, it does not make sense, we first have to instill the values and the awareness and then we need to do something, we can start with small things but first we have to educate, and the campaigns should start from the university and the university should realize it. It needs give more importance to the Environment Office and start to achieve that in all centers this should be seen as something necessary and create a real demand."

More generally, values also emerge as a central theme in the interviews and focus groups carried out in the green energy company. This is reflected in a relatively high frequency of valuerelated thematic codes, all pointing out that working in such a company affects the employees overall value priorities in a proenvironmental direction. This trend is well exemplified by the frequency of quotes such such as "Working in this company sensitizes people awareness of environmental issues" or "Working in this company strengthens individual proenvironmental behaviors". Respondents clearly acknowledge this aspect, and feel the need to present themselves and define their identity as "green", in particular in relation to energy saving: 
Table 1

Main contextual, organizational and individual barriers and drivers to pro-environmental and energy-related behaviors at work (Italian energy company).

\begin{tabular}{|c|c|c|}
\hline & Barriers & Drivers \\
\hline Contextual & $\begin{array}{l}\text { - Instable incentive policies (national level) } \\
\text { - Inefficient infrastructures (transport system) }\end{array}$ & $\begin{array}{l}\text { - External market } \\
\text { - Political regulations (European level) }\end{array}$ \\
\hline Organizational & $\begin{array}{l}\text { - Contrast between organizational mission and actual situation of the } \\
\text { workplace setting }\end{array}$ & $\begin{array}{l}\text { - Commitment to energy conservation as part of the values of the } \\
\text { whole company } \\
\text { - Efficient internal communication contribute to learning appropriate } \\
\text { behaviors }\end{array}$ \\
\hline Individual & $\begin{array}{l}\text { - Pay-off for energy-reduction: unsustainable behavior at work has no } \\
\text { direct financial consequences }\end{array}$ & - Employees taking active role for green organizational policies \\
\hline
\end{tabular}

Table 2

Main contextual, organizational and individual barriers and drivers to pro-environmental and energy-related behaviors at work (Spanish public University).

\begin{tabular}{|c|c|c|}
\hline & Barriers & Drivers \\
\hline Contextual & $\begin{array}{l}\text { - Larger cultural context perceived as unsupportive } \\
\text { - Economic crisis (high costs of technological change) }\end{array}$ & $\begin{array}{l}\text { - Political regulations (European level) } \\
\text { - Education and awareness policies }\end{array}$ \\
\hline Organizational & $\begin{array}{l}\text { - Organizational culture focused on control of the physical presence of } \\
\text { workers } \\
\text { - Insufficient communication of pro-environmental policies }\end{array}$ & - Commitment to energy conservation from management sensi \\
\hline Individual & $\begin{array}{l}\text { - Pay-off for energy-reduction: unsustainable behavior at work with } \\
\text { no direct financial consequences for individuals }\end{array}$ & $\begin{array}{l}\text { - Low-cost pro-environmental behaviors becoming part of the } \\
\text { habitual routines } \\
\text { - Feeling of individual responsibility }\end{array}$ \\
\hline
\end{tabular}

Participant 12 (Green energy company): "I feel the opportunity to work in this company... because you know the value of energy, our product, and you understand how many obstacles you have to make it real and usable. . .you also know economic and social costs. I am sure that we have more sensitiviness: is it something precious thing, or not?".

\section{Conclusions}

With the goal of understanding the main barriers to and drivers of pro-environmental and energy-related behavior in the workplace, we have undertaken a study of two large-scale organizations, characterized by a different focus and playing in very different contexts (the energy market vs. the public education sphere), but sharing a similar front-runner role as relevant actors in transitions to energetic sustainability. A qualitative study was carried out through a multi-method approach that included document analysis, in-depth interviews and focus groups with the aim to explore the interactions between organizational and individual factors in creating patterns of barriers and drivers for sustainable energy behaviors in the workplace. Results highlight a series of important conclusions for both researchers and practitioners striving to better understand how to promote pro-environmental behavior in the workplace. A summary of the main contextual, organizational and individual barriers and drivers is provided in Tables 1 and 2, for the Italian energy company and for the Spanish public University, respectively. As can bee seen, some of the drivers and barriers emerge in both organizations .

The analysis of both organizations has revealed that although organizations express a public commitment to sustainability, this does not always clearly translate into effective behavioral support strategies. Although employees choices might be motivated by a set of values that is part of either the organizational core business or social responsibility strategy, other factors play a more important role into how this commitment gets translated into everyday behavioral patterns. In spite of the fact that organizations belonged to different cultural contexts, we found that external contextual elements do not seem to be supportive of pro-environmental behavior, with the exception of European and, partly, national regulations. Organizations also might be reluctant to become frontrunners in transitions to sustainability, in the absence of such a certain and stable supportive context. Policy can play a bigger role in creating an external context in which organizations that assume the role of frontrunners in promoting pro-environmental behavior are adequately rewarded, and guaranteed about stable contextual conditions, especially in the domain of energy production and consumption.

Organizations are embedded in a cultural, social and economic environment that has an influence on many organizational aspects. It has been argued that the external environment places a series of institutional pressures on organizations, which can be regulatory, normative and socio-cultural, and that organizational culture is a response to these pressures in ways that have proven minimally adaptive over time [43]. Our results suggest that these types of pressures do influence environmental policy and its degree of implementation, both in a public university, and in a large-scale private company. These kind of "deep" assumptions around the relationship between economic and environmental objectives form part of the organizational culture, as Schein has argued (2010). Changing the organizational culture toward an environmental one would therefore require a change in the underlying beliefs about the interdependence of economic and environmental goals for an organization [36].

A second important finding of this study refers to the role of organizational social norms in the promotion of pro-environmental and energy-related behavior. Visibility of pro-environmental values through concrete manifestations such as regulations, policies, interventions, language and displayed behavior of leaders is a pre-condition for the generation of a shared perception of organizational support, which is the basis of organizational climate. Previous research provided evidence for a strong link between this latter variable and pro-environmental behavior [43]. These same studies have also suggested that individual perceptions of the organizational context play a role, such as the perceptions of whether the organization is committed with sustainable values and practices, as well as the perceptions of othersí behaviors and expectations, 
especially leaders [42]. Three different mechanisms have been proposed for the influence of leaders on workers, as direct motivators of ecological initiatives in the workplace: through motivational appeals [12]; through their role as relevant others and thus conveying social norms; through conveying visible support to employees already carrying out pro-environmental initiatives, thus motivating others to follow [39].

The way responsibility is attributed in an organization is also relevant, as it can constitute a motivation or a deterrent for action. Although the leadership of any organization has an important role in structuring the environment in which sustainable behaviors take place, workers can have a key role in transitions to sustainable organizations in two directions: by complying with environmental policies and responding to behavioral incentives on the one hand, and by going beyond compliance to promote and carry forward pro-environmental initiatives that contribute to the better overall organizational performance. For both types of pro-environmental action, organizational involvement and responsibility attribution are likely to play a key role. Our findings indicate that employees attribute an important part of the responsibility to the organization in designing the right context and providing the facilities for pro-environmental behaviors to take place, but at the same time they consider the lack of environmental awareness and education to be among the main barriers to sustainable energy behaviors at the individual level.

Finally, results of this study show that in order to become active promoters of pro-environmental behavior, certain types of organizations (especially those that can play a significant "stakeholder" role in energy efficiency efforts) need to become autonomypromoting contexts, and consequently change the implicit contract that regulates the relationships between employer and employee [46]. Changes toward autonomy-supporting contexts goes beyond traditional policies for organizational sustainability, generally conceptualized as a necessary part of the corporate social responsibility of organizations [44] or, more recently, as part of the core philosophy of organizations, besides profit and people [21]. Taken together, research on pro-environmental behavior in organizations tends to adopt a perspective of workers as rather passive recipients of organizational policy, influenced by systems of incentives to act in a certain way. However, given adequate contexts, workers can easily become also active promoters of sustainable transitions within organizations: the workplace is increasingly becoming a place in which individuals search for meaningful impact beyond making a living. Our results, taken together, show that by promoting autonomy-supporting contexts and an organizational culture that treats workers' input as a valuable and necessary part of the organizational performance, both public organizations and private companies have a chance to create an environment that not only encourages compliance with environmental policy, but also promotes active citizenship and engagement in organizational change, through the learning of new, and greener, lifestyles. As also other contributions in this volume suggest (e.g., [34,66]) promoting active citizenship and autonomy of single individuals, groups and communities will be a crucial challenge for energy transitions in the larger society, in the coming decades.

\section{Funding}

The research leading to these results has received funding from the European Union FP7 ENV.2010.4.2.3-1 grant agreement no 265155.

\section{Acknowledgments}

The authors are grateful to all the managers and leaders in the two case study organizations and to their colleagues and teams for the support in the data collection and participants recruiting process for this research.

\section{References}

[1] L.M. Anderson, T.S. Bateman, Individual environmental initiative: championing natural environmental issues in US business organizations, Acad. Manag. J. 43 (2000) 548-570.

[2] K. Araújo, The emerging field of energy transitions: progress, challenges, and opportunities, Energy Res. Soc. Sci. 1 (2014) 112-121.

[3] M. Birks, J. Mills, Grounded Theory: A Practical Guide, Sage, London, 2011.

[4] V. Blok, R. Wesselink, O. Studynka, R. Kemp, Encouraging sustainability in the workplace: a survey of the pro-environmental behavior of university employees, J. Clean. Prod. 106 (2015) 55-67.

[5] V. Braun, V. Clark, Using thematic analysis in psychology, Qual. Res. Psychol. 3 (2006) 77-101.

[6] K.A. Brekke, K. Nyborg, Attracting responsible employees: green production as labor market screening, Res. Energy Econ. 30 (2008) 509-526.

[7] G. Bridge, S. Bouzarovski, M. Bradshaw, N. Eyre, Geographies of energy transition: space, place and the low-carbon economy, Energy Policy 53 (2013) $331-340$.

[8] J. Brockner, E.T. Higgins, Regulatory focus theory: implications for the study of emotions at work, Organ. Behav. Hum. Decis. Process. 86 (2001) 35-66.

[9] S. Brondi, A. Armenti, C. Cottone, B.M. Mazzara, M. Sarrica, Parliamentary and press discourses on sustainable energy in Italy: no more hard paths, not yet soft paths, Energy Res. Soc. Sci. 2 (2014) 38-48.

[10] A. Brown, S. Kirpal, F. Rauner (Eds.), Identities at Work, Technical and Vocational Education and Training Series: Issues, Concerns and Prospects, vol. 5, Springer, Dordrecht, 2007.

[11] T. Busch, R. Bauer, M. Orlitzky, Sustainable development and financial markets old paths and new avenues, Bus. Soc. (2015), http://dx.doi.org/10. $1177 / 0007650315570701$.

[12] A.R. Carrico, M. Riemer, Motivating energy conservation in the workplace: an evaluation of the use of group-level feedback and peer education, J. Environ. Psychol. 31 (2011) 1-13.

[13] G. Carrus, M. Bonnes, F. Fornara, P. Passafaro, G. Tronu, Planned behavior and local norms: an analysis of the space-based aspects of normative ecological behavior, Cogn. Process. 10 (2009) 198-200.

[14] K. Charmaz, 'Discovering'chronic illness: using grounded theory, Soc. Sci. Med. 30 (1990) 1161-1172.

[15] R.B. Cialdini, Descriptive social norms as under-appreciated sources of social control, Psychometrika 72 (2007) 263-268.

[16] E.B. Craddock, A.H. Huffman, J.B. Henning, Taming the dragon: how industrial-organizational psychologists can break barriers to green business, Ind. Organ. Psychol. 5 (2012) 484-487.

[18] M.A. Delmas, S. Pekovic, Environmental standards and labor productivity: understanding the mechanisms that sustain sustainability, J. Organ. Behav. 34 (2013) 230-252

[19] T. Dietz, G.T. Gardner, J. Gilligan, P.C. Stern, M.P. Vandenbergh, Household actions can provide a behavioral wedge to rapidly reduce US carbon emissions, Proc. Natl. Acad. Sci. USA 106 (2009) 18452-18456.

[20] L. Di Lucia, K. Ericsson, Low-carbon district heating in Sweden-examining a successful energy transition, Energy Res. Soc. Sci. 4 (2014) 10-20.

[21] J. Elkington, Cannibals with Forks. The Triple Bottom Line of 21st Century Capstone, Oxford, 1997.

[23] N. Frantzeskaki, H. de Haan, Transitions: two steps from theory to policy, Futures 41 (2009) 593-606.

[24] R.W. Fri, M.L. Savitz, Rethinking energy innovation and social science, Energy Res. Soc. Sci. 1 (2014) 183-187.

[25] G.T. Gardner, P.C. Stern, Environmental Problems and Human Behaviour, 2nd edition, Pearson, Boston, 2002.

[26] B.G. Glaser, A.L. Strauss, The Discovery of Grounded Theory: Strategies for Qualitative Research, Aldine, Chicago, 1967

[27] M. Greaves, L.D. Zibarras, C. Stride, Using the theory of planned behavior to explore environmental behavioral intentions in the workplace, J. Environ. Psychol. 34 (2013) 109-120.

[28] D.W. Greening, D.B. Turban, Corporate social performance as a competitive advantage in attracting a quality workforce, Bus. Soc. 39 (2000) $254-280$.

[29] G. Grolleau, N. Mzoughi, S. Pekovic, Green not (only) for profit: an empirical examination of the effect of environmental-related standards on employees recruitment, Res. Energy Econ. 34 (2012) 74-92.

[30] N.J. Goldstein, R.B. Cialdini, V. Griskevisius, A room with a viewpoint: using social norms to motivate environmental conservation in hotels, J. Consum. Res. 35 (2008) 472-482.

[31] R.F. Hirsh, C.F. Jones, History's contributions to energy research and policy, Energy Res. Soc. Sci. 1 (2014) 106-111.

[32] A. Homburg, A. Stolberg, Explaining pro-environmental behavior with a cognitive theory of stress, J. Environ. Psychol. 26 (2006) 1-14.

[33] J.C. Hourcade, R. Crassous, Low-carbon societies: a challenging transition for an attractive future, Clim. Policy 8 (2008) 607-612.

[34] B.J. Kalkbrenner, J. Roosen, Citizens' willingness to participate in local renewable energy projects: the role of community and trust in Germany, Energy Res. Soc. Sci. (2016) (in press this volume, 2016). 
[35] W. Kempton, J.M. Darley, P.C. Stern, Psychological research for the new energy problems: strategies and opportunities, Am. Psychol. 47 (1992) 1213.

[36] M.K. Linnenluecke, A. Griffiths, Corporate sustainability and organizational culture, J. World Bus. 45 (2010) 357-366.

[37] S.H. Lo, G.J.Y. Peters, G. Kok, Energy-related behaviors in office buildings: a qualitative study on individual and organisational determinants, Appl. Psychol. 61 (2012) 227-249.

[38] S.H. Lo, G.J.Y. Peters, G. Kok, A review of determinants of and interventions for proenvironmental behaviors in organizations, J. Appl. Soc. Psychol. 42 (2012) 2933-2967.

[39] R. Lülfs, R. Hahn, Corporate greening beyond formal programs, initiatives: and systems: a conceptual model for voluntary pro-environmental behavior of employees, Eur. Manag. Rev. 10 (2013) 83-98.

[40] C.J.H. Midden, F.G. Kaiser, L.T. McCalley, Technology's four roles in understanding individuals conservation of natural resources, J. Soc. Issues 63 (2007) 155-174.

[41] C.A. Miller, J. Richter, J. O’Leary, Socio-energy systems design: a policy framework for energy transitions, Energy Res. Soc. Sci. 6 (2015) 29-40.

[42] T.A. Norton, H. Zacher, N.M. Ashkanasy, Organisational sustainability policies and employee green behaviour: the mediating role of work climate perceptions, J. Environ. Psychol. 38 (2014) 49-54.

[43] T.A. Norton, S.L. Parker, H. Zacher, N.M. Ashkanasy, Employee green behavior: a theoretical framework, multilevel review, and future research agenda, Organ. Environ. 28 (2015) 103-125.

[44] D.S. Ones, S. Dilchert, Environmental sustainability at work: a call to action, Ind. Organ. Psychol. 5 (2012) 444-466

[45] S. Oskamp, Psychological contributions to achieving an ecologically sustainable future for humanity, J. Soc. Issues 56 (2000) 373-390.

[46] P. Paillé, J.H. Mejía-Morelos, Antecedents of pro-environmental behaviours a work: the moderating influence of psychological contract breach, J. Environ. Psychol. 38 (2014) 124-131.

[47] P. Paillé, J.H. Mejía-Morelos, A. Marche-Paillé, C.C. Chen, Y. Chen, Corporate greening, exchange process among co-workers, and ethics of care: an empirical study on the determinants of pro-environmental behaviors at coworkers-level, J. Bus. Ethics (2015) 1-19, http://dx.doi.org/10.1007/s10551015-2537-0.

[48] N. Pidgeon, K. Henwood, Using grounded theory in psychological research, in: N. Hayes (Ed.), Doing Qualitative Analysis in Psychology, Psychology Press, Hove, UK, 1997, pp. 245-273.

[49] C.A. Ramus, U. Steger, The roles of supervisory support behaviors and environmental policy in employee ecoinitiatives at leading-edge European companies, Acad. Manag. J. 43 (2000) 605-626.

[50] J.L. Robertson, J. Barling, Greening organizations through leaders' influence on employees' pro-environmental behaviors, J. Organ. Behav. 34 (2013) 176-194
[51] M.V. Russo, P.A. Fouts, A resource-based perspective on corporate environmental performance and profitability, Acad. Manag. J. 40 (1997) 534-559.

[52] G.W. Ryan, H. Russell Bernard, Techniques to identify themes, Field Methods 15 (2003) 85-109.

[53] M. Sarrica, S. Brondi, P. Cottone, B.M. Mazzara, One, no one, one hundred thousand energy transitions in Europe: the quest for a cultural approach, Energy Res. Soc. Sci. (2016) (in press this volume, 2016).

[54] C.A. Scherbaum, P.M. Popovich, S. Finlinson, Exploring individual-level factors related to employee energy-conservation behaviors at work, J. Appl. Soc. Psychol. 38 (2008) 818-835.

[55] F.W. Siero, A.B. Bakker, G.B. Dekker, M.T. Van Den Burg, Changing organizational energy consumption behaviour through comparative feedback, J. Environ. Psychol. 16 (1996) 235-246.

[56] S. Siero, M. Boon, G. Kok, F. Siero, Modification of driving behavior in a large transport organization: a field experiment, J. Appl. Psychol. 74 (1989) 417-423.

[57] S. Sorrell, J. Dimitropoulos, The rebound effect: microeconomic definitions, limitations and extensions, Ecol. Econ. 65 (2008) 636-649.

[58] B. Sovacool, How long will it take? Conceptualizing the temporal dynamics of energy transitions, Energy Res. Soc. Sci. (2016) (in press this volume, 2016).

[59] B.K. Sovacool, What are we doing here? Analyzing fifteen years of energy scholarship and proposing a social science research agenda, Energy Res. Soc Sci. 1 (2014) 1-29.

[60] M. Starik, A.A. Marcus, Introduction to the special research forum on the management of organizations in the natural environment: a field emerging from multiple paths, with many challenges ahead, Acad. Manag. J. 43 (2000) 539-547.

[61] L. Steg, C. Vlek, Encouraging pro-environmental behaviour: an integrative review and research agenda, J. Environ. Psychol. 29 (2009) 309-317.

[62] P.C. Stern, Psychological dimensions of global environmental change, Annu. Rev. Psychol. 43 (1992) 269-302.

[63] P. Stern, Toward a coherent theory of environmentally significant behavior, J. Soc. Issues 56 (2000) 407-424.

[64] T.L. Tudor, S.W. Barr, A.W. Gilg, A novel conceptual framework for examining environmental behavior in large organizations a case study of the Cornwall National Health Service (NHS) in the United Kingdom, Environ. Behav. 40 (2008) 426-445.

[65] D.B. Turban, D.W. Greening, Corporate social performance and organizational attractiveness to prospective employees, Acad. Manag. J. 40 (1997) 658-672.

[67] E.U. Weber, P.C. Stern, Public understanding of climate change in the United States. Am, Psychol. 66 (2011) 315-328.

[68] L. Andersson, S.E. Jackson, S.V. Russell, Greening organizational behavior: an introduction to the special issue, J. Organ. Behav. 34 (2013) 151-155.

[69] P.M. Blau, Exchange and Power in Social Life, Wiley, New York, 1964 